\title{
BIOGENERIC PRODUCTS: AN INTRODUCTION
}

\author{
IVy Louis*
}

The biotech industry is 30 years old and many patent expirations are just a few years away. Normally generic competition would be maneuvering in, developing and testing substitutes in anticipation of the opened door and this is what brings the term biogenerics. Indian biotech sector has witnessed immense growth in providing the generic versions of the patented molecules that are available the world over, but have not been able to reach the shores of regulated markets, as the process of approving the generic versions of the biotech molecules is long and arduous. This paper discusses a brief of the fundamentals about biogenerics and aims at increasing the understanding of the terms used by the research/development and/or the manufacturing firms.

\section{What are Biogenerics or Generics?}

A generic drug (pl. generic drugs, short: generics) is a drug which is bioequivalent to a brand name drug with respect to pharmacokinetic and pharmacodynamic properties. These drugs are usually sold at a lower price than the brand name drug. Generic medicines must contain the same active ingredient at the same strength as the "innovator" brand, be bioequivalent, and are required to meet the same pharmacopoeial requirements for the preparation. By extension, therefore, generics are assumed to be identical in dose, strength, route of administration, safety, efficacy, and intended use.

* Business Manager- Technology Operations, Bioprocess Division, Millipore India, Bangalore. 
Thus, biogenerics are generic forms of biopharmaceuticals -molecules developed using biological processes, usually through modern biotechnology activity. Generic chemical pharmaceuticals can be defined as those molecules which, when compared with the originator product:
* have essentially similar activity
* are chemically identical to their branded counterparts
* are bioequivalent and
- achieve market authorization through an abbreviated procedure (ANDA) following patent expiry.

\section{Reasons for Cheaper Price}

The principal reason for the reduced price of generic medicines is that these companies incur less costs in creating the generic drug and are therefore able to - offer a lower price and still maintain profitability. Manufacturers of generic drugs are mainly able to avoid the following three costs that brand name pharmaceutical companies incur:

(1) costs associated with the research and development of the drug; Generic manufacturers do not incur the cost of drug discovery and instead reverse-engineer existing brand name drugs to allow them to manufacture bioequivalent versions. (2) costs associated obtaining regulatory approval (i.e. proving safety and efficacy of a drug); Generic manufacturers do not bear the burden of proving the safety and efficacy of the drugs through clinical trials - rather, generic manufacturers must prove the bioequivalance to the existing drugs. (3) marketing cost; Generic companies receive the large benefit of the marketing that goes into pushing the innovator drug.

\section{Patent Lifetime and Research Cost Issues}

Pharmaceutical companies may produce a generic drug when the patent expires on the innovator drug. Patent lifetime differs from country to country. The length of time before a patent expires varies for different drugs. Usually, there is no way to renew a patent after it expires. A new version of the drug with significant changes to the compound could be patented but this will require new clinical trials and will not prevent the generic versions of the original drug. Usually, generic drugs are much less expensive than the brand-name product. Some patients and physicians will 
hesitate to select these medications because of concerns about the quality of generic drugs. When a pharmaceutical company first markets a drug, it is usually under a patent that allows only the pharmaceutical company that developed the drug to sell it. This allows the company to recoup the cost of developing that particular drug. It costs on average around $\$ 800,000,000$ to develop and test a new drug before it is approved for use. After the patent on a drug expires, any pharmaceutical company can manufacture and sell that drug. Since the drug has already been tested and approved, the cost of simply manufacturing the drug will be a fraction of the original cost of testing and developing that particular drug. The brand-name drug companies have tended to litigate aggressively to extend patent protection on their medicines and keep generic versions off the market, a process referred to by critics as "evergreening." Generic drugs start out at first being fairly expensive, however the price of the generic product decreases as the rate of production increases.

\section{When can a Generic Drug be Produced?}

Generic drugs can be legally produced for drugs where: 1) the patent has expired, 2) the generic company certifies the brand company's patents are either invalid, unenforceable or will not be infringed, 3) for drugs which have never held patents, or 4) in countries where a patent(s) is/are not in force.

The expiration of a patent removes the monopoly of the patent holder on drug sales licensing. It is also becoming popular for the large pharmaceutical companies to preempt the expiry of their patent by producing their own generic product, or license their own product to be branded by generic companies. Thus, in some cases, the "generic" product is actually the brand product but inside a different box.

Enacted in 1984, the U.S. Drug Price Competition and Patent Term Restoration Act, informally known as the "Hatch-Waxman Act", standardized U.S. procedures for recognition of generic drugs. An applicant files an Abbreviated New Drug Application (or "ANDA") with the Food and Drug Administration (FDA) and seeks to demonstrate therapeutic equivalence to a specified, previously approved "reference listed drug." When an ANDA is approved, the FDA adds the drug to its Approved Drug Products list, also known as the "Orange Book", and annotates the list to show equivalence between the reference listed drug and the approved generic. The FDA also recognizes drugs using the same ingredients with different bioavailability and divides them into therapeutic equivalence groups.

The first step in assembling a list of potential biogeneric products is to examine the Food and Drug Administration agency's definitions and regulatory pathways for 
drugs and biologicals. Many names have been proposed to describe such products, including biogenerics, biosimilars, generic biologics, follow-on-biologics (FOB) and follow-on protein products (FOPP). Definitions vary, but most convey similar concepts. The Unites States Food and Drug Administration's (US FDA) definition of follow-on protein product is as "a protein product which is intended to be a similar version or duplicate of an already approved or licensed protein product."

Generic drug applications are termed "abbreviated" because they are generally not required to include preclinical (animal) and clinical (human) data to establish safety and effectiveness. Instead, generic applicants must scientifically demonstrate that their product is bioequivalent (i.e., performs in the same manner as the innovator drug). One way scientists demonstrate bioequivalence is to measure the time it takes the generic drug to reach the bloodstream in 24 to 36 healthy volunteers. This gives them the rate of absorption, or bioavailability, of the generic drug, which they can then compare to that of the innovator drug. The generic version must deliver the same amount of active ingredients into a patient's bloodstream in the same amount of time as the innovator drug. Using bioequivalence as the basis for approving generic copies of drug products was established by the Hatch-Waxman Act. This Act expedites the availability of less costly generic drugs by permitting FDA to approve applications to market generic versions of brand-name drugs without conducting costly and duplicative clinical trials. At the same time, the brand-name companies can apply for up to five additional years longer patent protection for the new medicines they developed to make up for time lost while their products were going through FDA's approval process. Brand-name drugs are subject to the same bioequivalence tests as generics upon reformulation. (Bioequivalence is a term in pharmacokinetics used to assess the expected in vivo biological equivalence of two proprietary preparations of a drug. If two products are said to be bioequivalent it means that they would be expected to be, for all intents and purposes, the same.)

\section{The Regulatory Maze and Pathway to Approvals}

The Food and Drug.Administration says it's difficult to make generic versions of biotechnologically processed/manufactured drugs because they are based on complex biological organisms, rather than simple molecular compounds like the more traditional pharmaceutical drugs. Manufacturers in many countries including India are already producing them and estimates hold that by 2010 some $\$ 20$ billion worth of biogenerics will begin to become available. 
In the USA the Food and Drug Administration (FDA) is responsible for making sure that generic drugs are "safe and effective." The approval process for generic drugs began in the late 1960s. Generic drug manufacturers were required to prove that their formulation exhibits bioequivalence to the innovator product. Over the past several years there have been studies that have shown the effectivness and safety of some generic drugs. Generic drugs can save patients and insurance companies thousands of dollars supposedly without compromising the quality of care. The FDA must approve generic drugs just as innovator drugs must be approved. Bioequivalence, however, does not mean that generic drugs are exactly the same as their innovator product counterparts, as chemical differences do exist.

Approvals for bringing out biogenerics are made when biopharmaceutical organizations submit proposals to the regulators for marketing approval of their molecules, via an abbreviated route. The regulators indicate if they are satisfied with their approaches and proceed to approve of these FOPP's (Follow on Protein Products) or FOB's (Follow on Biologics). Big Pharma companies are scrambling to make up for multi-billion dollar sales vacuums as key blockbusters - like the Merck cholesterol-cutter Zocor and the Pfizer antidepressant Zoloft - run out of patent protection. When this happens, generic drug makers produce versions at severely reduced prices, and then revenue plunges. The biotech sector isn't at that point yet. Analysts say that big biotechs, like Amgen and Genentech are unlikely to feel the pinch of immediate generic pressure because the patent expirations on their biggest blockbusters are years away. Analysts believe that the first biotech blockbuster to face patent expiration will be Epogen, an anemia treatment for dialysis patients from Amgen, the world's biggest biotech. The patent is expected to run out in 2011. A new drug can take years to get approval from the FDA. So any company looking to make a biogeneric substitute for Epogen would have to start the process about now.

But beyond the time factor, there are technical issues for biogenerics as well. It's probably not as big a threat to biotech as the normal generic industry is to pharma also; biotech drugs require intensive work from biogenerics companies in order to copy them. Unlike the generic pharmaceutical industry, biogeneric companies may have to run expensive and time-consuming clinical trials. There is no way to 100 percent accurately reproduce the host that these compounds grow off of and therefore it can never be functionally identical. The only way for a biogeneric company to produce an exact copy of a brand-name biotech drugs, is to sneak into the factory and to steal the host, which is unethical and illegal. 


\section{Day Generic Drug Exclusivity}

The US FDA offers a 180 day exclusivity period to generic drug manufacturers in specific cases. During this period only one (or sometimes a few) generic manufacturers can produce the generic version of a drug. This exclusivity period is only used when a generic manufacturer argues that a patent is invalid or is not violated in the generic production of a drug, and the period acts as a reward for the generic manufacturer who is willing to risk liability in court and the cost of patent court litigation. There is often contention around these 180 day exclusivity periods because a generic producer does not have to produce the drug during this period and can file an application first to prevent other generic producers from selling the drug.

Large pharmaceutical companies often spend thousands of dollars protecting their patents from generic competition. Apart from lifigation, companies use other methods such as reformulation or licensing a subsidiary (or another company) to sell generics under the original patent. Generics sold under license from the patent holder are known as authorized generics; they are not affected by the 180 day exclusivity period as they fall under the patent holder's original drug application.

A prime example of how this works is Simvastatin (Zocor), a popular drug created and manufactured by U.S. based pharmaceutical Merck \& Co., which lost its US patent protection on June 23, 2006. India-based Ranbaxy Laboratories (at the 80$\mathrm{mg}$ strength) and Israel-based Teva Pharmaceutical Industries (at all other strengths) received 180 day exclusivity periods for Simvastatin; due to Zocor's popularity, both companies began marketing their products immediately after the patent expired.

\section{What are the characteristics of a successful biogeneric product?}

For biogeneric products to be successful there should be:

- No IP barrier, therefore biological products facing patent expiration all have the potential to become successful biogenerics

- The right market characteristics - the questions which must be asked here are:

- Is the market big enough?

- Is there potential to make a worthwhile profit in this area? 
- What is the competition like?

- Are there many entrenched players in the market already?

- Are there many other biogenerics players entering this market?

- Are biogeneric players already producing this product outside the Westem markets?

These drugs propel the growth of markets when the affordability of patented molecules is out of reach for the common man and biopharmaceutical industries rush into such domains to make healthcare affordable and available.

\section{So, when will Biogenerics reach the markets}

The first companies will take time to gain entry to the market; but once this has happened, the biogenerics market will evolve more rapidly. The biogenerics market will be different from the generics market. There will be fewer players due to consolidation brought about by complex manufacturing processes, difficulties in gaining approval and the high cost of successfully marketing products. It will be three to four years before the limited numbers of main players have their first products on the market. Most consider that these main players will have the market to themselves for the first few years and it is likely to be several years before generic competition has a significant effect on the market, possibly longer than most potential players acknowledge in their public statements. The public authorities might be expected to promote biogenerics, encouraging price competition to ease the burden of payment for biotechnology-derived treatments. This, however, is unlikely to be the strategy that the early biogeneric entrants will wish to adopt, having had to invest significantly to:

- bring their products to market

- gain market acceptance for their products.

The investment required is substantially more than was envisaged just ten to fifteen years ago, when companies began to consider their involvement in this area. The general view is that the US will represent a harder market for biogenerics to access. The big pharma lobby is very strong, with patents lasting longer. The situation is becoming increasingly legalistic and the biotechnology innovators are likely to challenge every change in legislation, essentially employing effective delaying tactics. The FDA has announced that the 505(b)(2) pathway is permissible for approval of 'generic' versions of biological products that were originally approved under an 
NDA (New Drug Application) as opposed to a biologics license application (BLA). Many generic companies are advocating a 505 (b)(2)-like approach to approval of generic versions of more complex biologics originally approved under BLAs. Such a system might resemble certain aspects of the recent guidance by the European Agency termed EMEA (Directive 2003/63/EC, June 2003).

The complexity and details of a stringent rule-based system such as that seen in the US means that it is always open to legal challenges, which will inevitably delay changes in the regulatory framework, although many current developments suggest that the process of having biogenerics made available to the common man is on a fast-track. 